\title{
Rare localized extralobar sequestration with congenital cystic adenomatoid malformation: a case report
}

\author{
Satoshi Nagasaka ${ }^{1 *} \mathbb{D}$, Satsuki Kina', Yoshihito Arimoto ${ }^{1}$, Fumi Yokote' ${ }^{1}$ Tsuyoshi Uchida ${ }^{2}$ and Hirochika Matsubara ${ }^{2}$
}

\begin{abstract}
Extralobar sequestrations constitute a rare form of congenital pulmonary airway malformations that are difficult to diagnose. Here, we report a rare case of a localized extralobar sequestration in the right superior portion of the mediastinum accompanied by congenital cystic adenomatoid malformation.

A 19-year-old man presented with a right upper mediastinal mass that was detected using chest radiography, had a history of left spontaneous pneumothorax, and had undergone a bullectomy 4 years previously.

The initial diagnosis included a mature teratoma and a bronchogenic cyst in the mediastinum; however, the presence of a cystic mass in the right upper lobe of the lung prompted further examination. A preoperative diagnosis of extralobular sequestration was finally determined using contrast-enhanced computed tomography. The aberrant artery was connected to the brachiocephalic artery, and its drainage vein was connected to the right pulmonary artery, uniquely behind the pericardium. Despite the unique location, right mediastinal extralobular sequestration with a congenital cystic adenomatoid malformation in the right upper lobe was confirmed pathologically. Examination of contrast-enhanced chest computed tomography (CT) and three-dimensional computed tomography images enabled a correct diagnosis. It is very important for surgeons to correctly diagnose and identify an aberrant artery and drainage vein to prevent uncontrolled hemorrhage.
\end{abstract}

Keywords: Congenital cystic adenomatoid malformation, Extralobar pulmonary sequestration, Right mediastinal mass, Mediastinal extralobar sequestration

\section{Background}

Pulmonary sequestrations in adults are a rare congenital anomaly. An extralobar sequestration (ELS) is the least common of all congenital pulmonary airway malformations. It is often located in the left lower lobe and diaphragm and receives its blood supply from systemic arteries, whereas its venous drainage occurs via a systemic vein. The atypical location makes it difficult to diagnose this condition correctly. Here, we report a unique case of right mediastinal ELS that was diagnosed preoperatively and successfully treated surgically.

\footnotetext{
* Correspondence: snagasak@hosp.ncgm.go.jp

${ }^{1}$ General Thoracic Surgery, National Center for Global Health and Medicine,

1-21-1 Toyama, Shinjuku-ku, Tokyo 162-8655, Japan

Full list of author information is available at the end of the article
}

\section{Case presentation}

A 19-year-old man was referred to our hospital for a right upper mediastinal mass that was detected on chest radiographic examination. The patient had no symptoms; however, he had a medical history of left spontaneous pneumothorax and had undergone a bullectomy 4 years previously. Plain chest computed tomography (CT) revealed a multilobulated cystic mass that was sharply defined and triangular shaped, measuring $52 \times$ $31 \times 25 \mathrm{~mm}^{3}$ with calcification, and surrounded by the right innominate vein, the right upper lobe of the lung, and the trachea (Fig. 1a). Initial diagnoses revealed a mature teratoma and a bronchogenic cyst. On the other hand, localized multicystic lesions were detected in the right anterior segment of the lung, which were completely separated from the mediastinal tumor (Fig. 1b). Despite the patient's medical history, considering his age, the lesion on the inner side of the lung suggested 


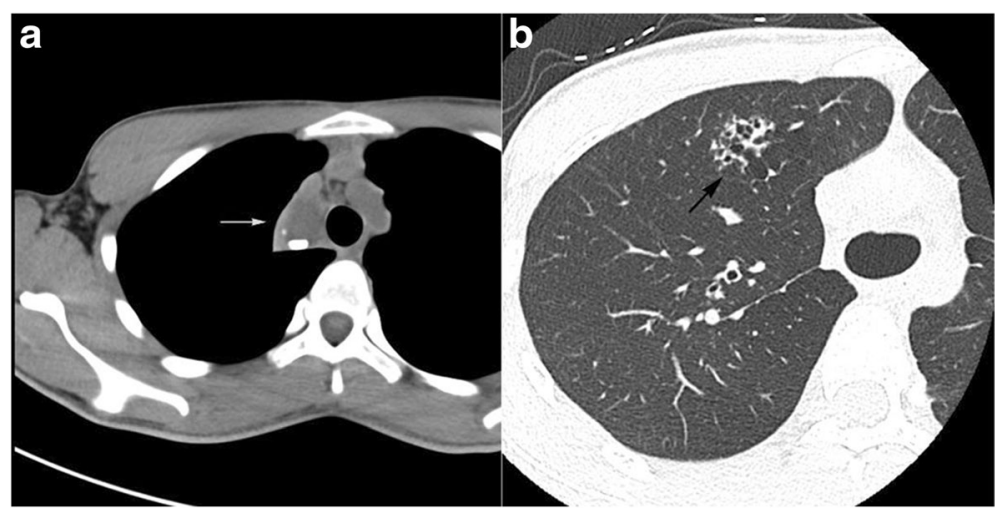

Fig. 1 a Plain chest computed tomography image (axial) shows a right anterior mediastinal cystic mass with calcification (arrow). b Chest computed tomography image shows a cystic lesion in the right anterior segment (arrow)

not only the impossibility of a pneumothorax but also other potential underlying diseases.

Contrast-enhanced chest CT revealed strong enhancement around the tumor and an aberrant artery originating from the brachiocephalic artery and draining into the right main pulmonary artery behind the pericardium. Despite the unique location, ELS with a congenital cystic adenomatoid malformation (CCAM) of the right upper lobe was strongly suspected.

The tumor was examined in detail using threedimensional CT (Fig. 2a). No other regions with associated abnormalities were detected on further examination, except for the cystic lesion in the right anterior segment.

A sternotomy was performed, and a soft heterogeneous mass with its own visceral pleura was detected surrounding the right and left innominate veins. Arteries originating from the brachiocephalic artery were identified, which were also visible on three-dimensional CT. The drainage vein was followed in the pericardium and ligated at the top of the right main pulmonary artery behind the dorsal pericardium (Fig. 2b). The mediastinal mass shrank after ligating the aberrant artery and the drainage vein. The cystic mediastinal tumor, which existed independently in the mediastinal pleura, was completely removed. In addition, the cystic mass in the right upper lobe was partially resected.

The macroscopic findings indicated a mediastinal mass that measured $48 \times 28 \times 23 \mathrm{~mm}^{3}$ and was encased within its own pleura. Histological findings revealed that the cyst wall was covered with ciliated columnar epithelium and surrounded by cartilage and bronchial glands. Hematoxylin and eosin staining showed that the tortuous artery was thick walled (Fig. 3a), and Elastica van Gieson staining identified elastic fibers in the intimal layer of the tortuous artery (Fig. 3b). These findings were compatible with ELS. The surgical specimen of the right anterior segment of the lung revealed that the wall of the cyst was lined by cuboidal and pseudostratified, respiratory-like epithelium (via hematoxylin and eosin staining), which led to

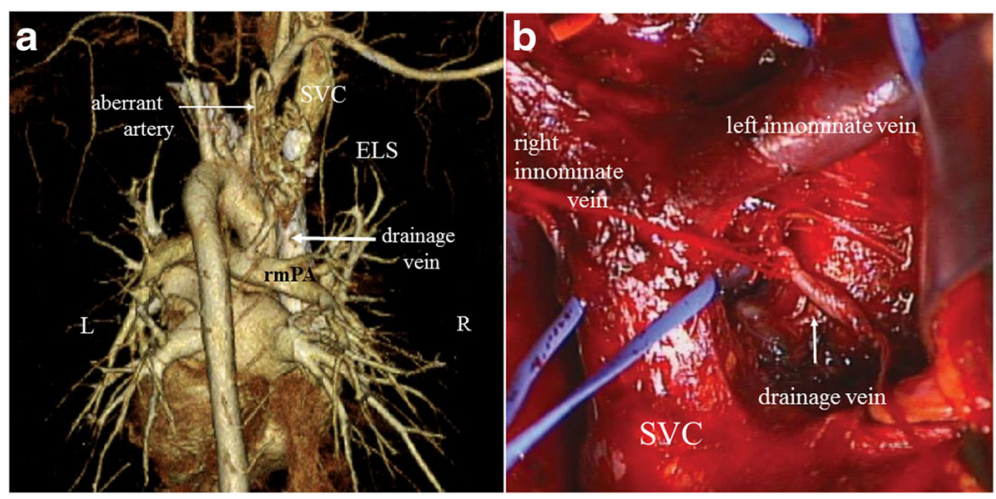

Fig. 2 a Three-dimensional reconstruction computed tomography image (posterior view) shows an aberrant artery branching out from the brachiocephalic trunk and the vein draining into the right pulmonary artery. $\mathbf{b}$ Intraoperative findings show the drainage vein (ligated; arrow) to the right pulmonary artery behind the dorsal pericardium (cut away) between the ascending aorta (pulled to the left side) and the superior vena cava(SVC). The right and left innominate veins and the SVC are taped. ELS extralobar sequestration, rmPA right main pulmonary artery, SVC superior vena cava 


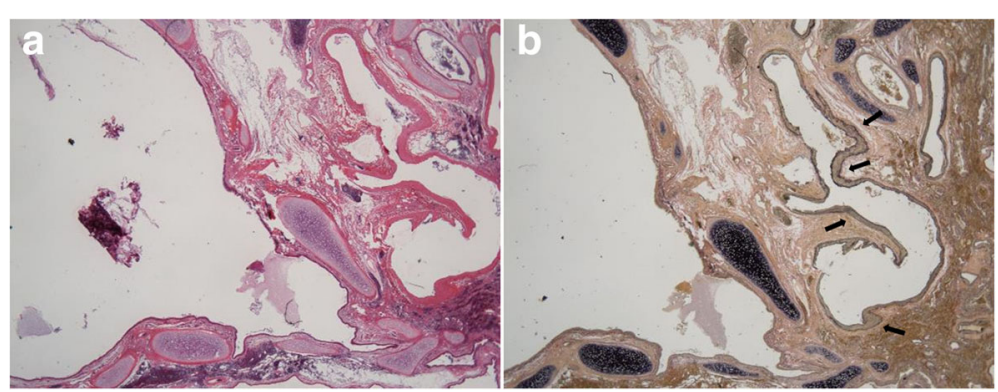

Fig. 3 a ELS. Histological findings reveal that the dilated cyst wall is covered with ciliated columnar epithelium, surrounded by cartilage and bronchial glands (hematoxylin and eosin staining, original magnification $\times 10$ ). b Elastica van Gieson staining showed elastic fibers in the intimal layer of the tortuous artery (arrow) (original magnification $\times 10$ )

the diagnosis of CCAM (Fig. 4). There was no evidence of malignancy in the ELS or CCAM.

In addition, the specimen of his previous pneumothorax showed normal bulla and blebs of the lung.

The patient's postoperative course was excellent. At the time of this report, he had been faring well with no postsurgical complications for 2 years.

\section{Conclusions}

In adult patients, pulmonary sequestrations are a rare congenital malformation of the lung. However, they are the second most common congenital lung anomaly in pediatric respiratory disease because they are typically diagnosed during childhood.

A pulmonary sequestration is a lung anomaly lacking normal tracheobronchial connections and supplied systemic arteries. Pulmonary sequestrations are divided into two types, intralobar sequestration (ILS) and ELS, based on whether the visceral pleura does or does not separate the normal lobe, respectively. An ELS is rare; they usually

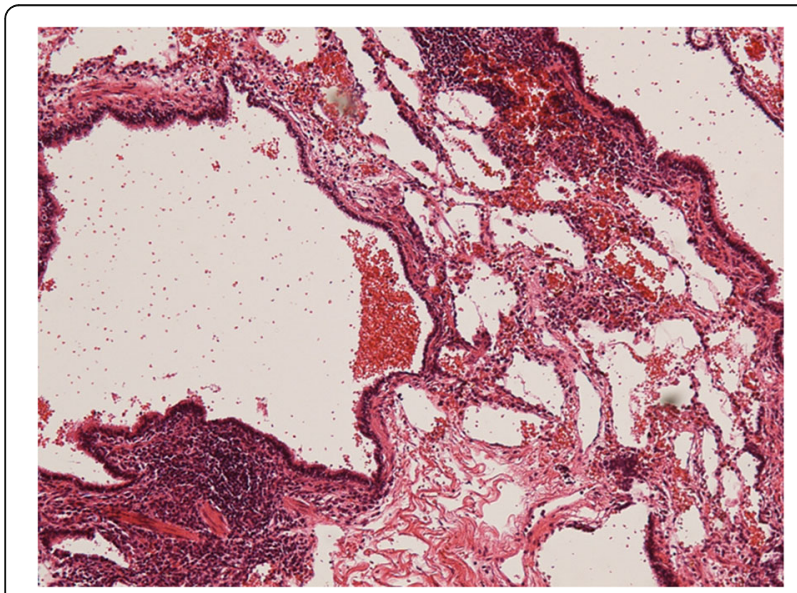

Fig. 4 CCAM. The surgical specimen of the lung shows that the wall of the cyst is lined with cuboidal and pseudostratified, respiratory-like epithelium with chronic inflammatory cell infiltration. (hematoxylin and eosin staining, original magnification $\times 40$ ) occur between the lower lobe of the lung and diaphragm and manifest with multiple anomalies. CCAM is also a rare developmental lung anomaly in adolescence and adulthood, but it is the most common congenital lung lesion in childhood examined by routine ultrasound. Stocker divided CCAM that are hamartomatous lesions comprising cystic and adenomatous elements into three major types based upon the size of the cysts and their cellular characteristics [1]. The present case involved type II CCAM comprising multiple cysts approximately $0.5 \mathrm{~cm}$ in diameter and solid areas that blended into adjacent normal tissue.

There are two major theories surrounding the source of lung anomalies: (1) they originate in the primitive foregut or lung bud and (2) they arise from the six pairs of aortic arches or venous radicals and their derivatives. Richard et al. [2] reported that proportion of ELS with frequently associated congenital cystic adenomatoid malformation type II was approximately $50 \%$. Recently, the pathogenesis of CCAM, LLS, ELS, and lobar emphysema has been thought to have common origins in different stages of lung development and at different sites and times, under the influence of airway obstruction [3, 4]. We considered co-existence of these two diseases in the present case seems not to be coincidental. Congenital cystic adenomatoid malformation was considered a premalignant lesion, and right upper lobectomy was preferable. Therefore, careful long-term follow-up was required.

Among 133 cases of ELS reported by Savic et al. [5], only two were located in the right mediastinum. An ELS of the right superior portion of the mediastinum may be rarer. The arterial supply is normally provided by branches of the aorta, and the venous drainage is normally routed through the systemic veins, usually the inferior vena cava or azygos-hemiazygos systems. However, in this patient, the venous return was unique. The drainage vein returned to the right main pulmonary artery behind the dorsal pericardium.

Savic et al. [5] reported that only six of 66 patients who underwent surgery for ELS had received a correct 
diagnosis. A unique location can make a preoperative diagnosis even more difficult. However, carefully interpreting contrast-enhanced chest CT and three-dimensional CT images can ensure a correct diagnosis. An accurate diagnosis results in a safe and easy operation. Physicians should take great care in diagnosing a mediastinal tumor, regardless of whether it is in an atypical location [6-8].

Savic et al. [5] reported five patients with an ILS who died because of uncontrolled bleeding of the aberrant artery. In patients with ELS, massive intraoperative bleeding due to injured aberrant vessels has been reported [9]. It is therefore very important to identify aberrant vessels to prevent an uncontrolled hemorrhage. It is very useful to examine contrast-enhanced chest CT images and to create a reconstructed three-dimensional CT image for safe surgery.

Pulmonary sequestration with CCAM has recently been reported more frequently in pediatric surgery [2-4]. Due to the presence of a mediastinal tumor with a cystic mass in the lung, a sequestration was also suspected in this patient.

\section{Abbreviations}

CCAM: Congenital cystic adenomatoid malformation; CT: Computed tomography; ELS: Extralobar sequestration

\section{Acknowledgements}

We thank Dr. Takashi Okafuji (Department of Radiology at the National Center for Global Health and Medicine, Tokyo, Japan) for the radiological assessment. We also thank Dr. Toshihiko lizuka (Department of Diagnostic Pathology at the National Center for Global Health and Medicine, Tokyo, Japan) for the histological assessment of the tumor.

\section{Funding}

None of the authors received any funding for this study.

\section{Authors' contributions}

SN is the corresponding author and revised the manuscript. SN, TU, FY, YA, and SK performed the operation. SN and HM designed and drafted the manuscript. SN, YA, and SK collected the imaging films and histological samples. All authors have read and approved the final version of the manuscript.

\section{Competing interests}

The authors declare that they have no competing interests

\section{Consent for publication}

Written informed consent was obtained from the patient for publication of this case report and any accompanying images. A copy of the written consent is available for review by the Editor-in-Chief of this journal.

\section{Ethics approval and consent to participate}

Not applicable.

\section{Declarations}

The authors had full control of the design of the study, methods used, outcome parameters, the results and analysis of data, and production of the written report.

\section{Publisher's Note}

Springer Nature remains neutral with regard to jurisdictional claims in published maps and institutional affiliations.

\section{Author details}

'General Thoracic Surgery, National Center for Global Health and Medicine, 1-21-1 Toyama, Shinjuku-ku, Tokyo 162-8655, Japan. ${ }^{2}$ Second Department of Surgery, University of Yamanashi Hospital, 1110, Shimokato, Chuo-shi,

Yamanashi 409-3898, Japan.

Received: 29 October 2016 Accepted: 14 March 2017

Published online: 21 March 2017

\section{References}

1. Stocker JT, Madewell JE, Drake RM. Congenital cystic adenomatoid malformation of the lung. Classification and morphologic spectrum. Hum Pathol. 1977;8(2):155-71.

2. Conran RM, Stocker JT. Extralobar sequestration with frequently associated congenital cystic adenomatoid malformation, type 2: report of 50 cases. Pediatr Dev Pathol. 1999;2:454-63.

3. Riedlinger WF, Vargas SO, Jennings RW, Estroff JA, Barnewolt CE, Lillehei CW, et al. Bronchial atresia is common to extralobar sequestration, intralobar sequestration, congenital cystic adenomatoid malformation, and lobar emphysema. Pediatr Dev Pathol. 2006;9:361-73.

4. McLean SE, Pfeifer JD, Siegel MJ, Jensen ER, Schuler PM, Hirsch R, et al. Congenital cystic adenomatoid malformation connected to an extralobar pulmonary sequestration in the contralateral chest: common origin? J Pediatr Surg. 2004:39:e13-7.

5. Savic B, Birtel FJ, Tholen W, Funke HD, Knoche R. Lung sequestration: report of seven cases and review of 540 published cases. Thorax. 1979;34:96-101.

6. Sippel JM, Ravichandran PS, Antonovic R, Holden WE. Extralobar pulmonary sequestration presenting as a mediastinal malignancy. Ann Thorac Surg. 1997;63:1169-71.

7. Shadmehr MB, Jamaati HR, Saidi B, Tehrai M, Arab M. Extralobar sequestration in anterior mediastinum with pericardial agenesis. Ann Thorac Surg. 2009;88:291-3.

8. Osaki T, Kodate M, Takagishi T, Nomi M, Murakami J, Yamamoto H. Unique extralobar sequestration with atypical location and aberrant vessels. Ann Thorac Surg. 2010:90:1711-2.

9. Datta G, Tambiah J, Rankin S, Herbert A, Lang-Lazdunski L. Atypical presentation of extralobar sequestration with absence of pericardium in an adult. J Thorac Cardiovasc Surg. 2006;132:1239-40.

\section{Submit your manuscript to a SpringerOpen ${ }^{\mathcal{O}}$ journal and benefit from:}

- Convenient online submission

- Rigorous peer review

- Immediate publication on acceptance

- Open access: articles freely available online

- High visibility within the field

- Retaining the copyright to your article

Submit your next manuscript at $\boldsymbol{s p r i n g e r o p e n . c o m ~}$ 